\title{
Correction to: Genome-wide assessment of DNA methylation in mouse oocytes reveals effects associated with in vitro growth, superovulation, and sexual maturity
}

Maria Desemparats Saenz-de-Juano ${ }^{1,2}$, Elena Ivanova ${ }^{3}$, Katy Billooye ${ }^{1}$, Anamaria-Cristina Herta', Johan Smitz ${ }^{1}$ Gavin Kelsey ${ }^{3,4}$ and Ellen Anckaert ${ }^{1 *}$

\section{Correction to: Clin Epigenet https://doi.org/10.1186/s13148-019-0794-y}

After publication of the original article [1], we were notified that:

the software used to create the figures has exported them wrong so they display incomplete. Below you can find the correct version of the figures:

The original article has been corrected.

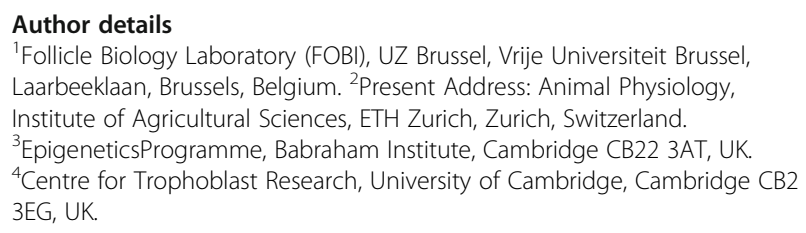

Published online: 27 January 2020

\section{Reference}

1. Saenz-de-Juano, et al. Genome-wide assessment of DNA methylation in mouse oocytes reveals effects associated with in vitro growth, superovulation, and sexual maturity. Clin Epigenet. 2019;11:197. https://doi. org/10.1186/s13148-019-0794-y.

The original article can be found online at https://doi.org/10.1186/s13148019-0794-y

* Correspondence: ellen.anckaert@uzbrussel.be

${ }^{1}$ Follicle Biology Laboratory (FOBI), UZ Brussel, Vrije Universiteit Brussel,

Laarbeeklaan, Brussels, Belgium

Full list of author information is available at the end of the article

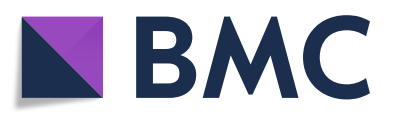

(อ) The Author(s). 2020 Open Access This article is distributed under the terms of the Creative Commons Attribution 4.0 International License (http://creativecommons.org/licenses/by/4.0/), which permits unrestricted use, distribution, and reproduction in any medium, provided you give appropriate credit to the original author(s) and the source, provide a link to the Creative Commons license, and indicate if changes were made. The Creative Commons Public Domain Dedication waiver (http://creativecommons.org/publicdomain/zero/1.0/) applies to the data made available in this article, unless otherwise stated. 
A

IFC: In vitro follicle culture
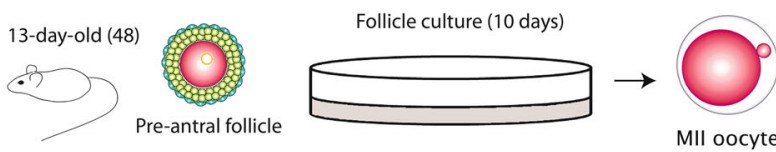

IFC

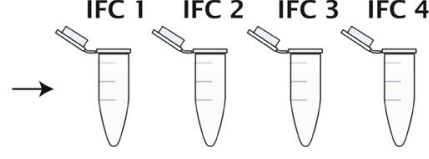

SO: Superovulation prepubertal

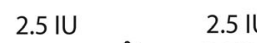

$\begin{array}{llll}104 & 158 & 152 & 72\end{array}$
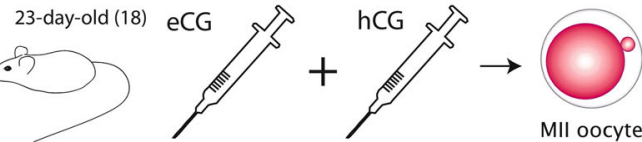

SO 1

SO 2 SO 3

SO 4 SO 5
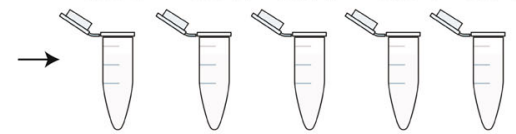

SOA: Superovulation adult
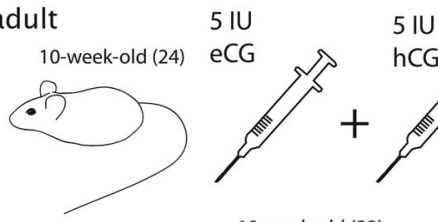

hCG

137

157

130

70
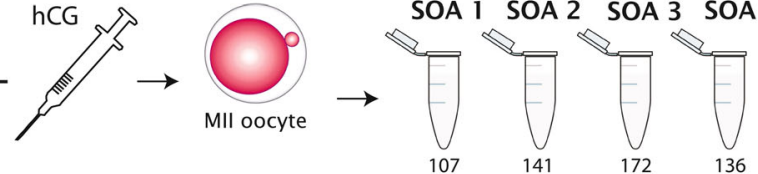

IV: In vivo natural ovulation
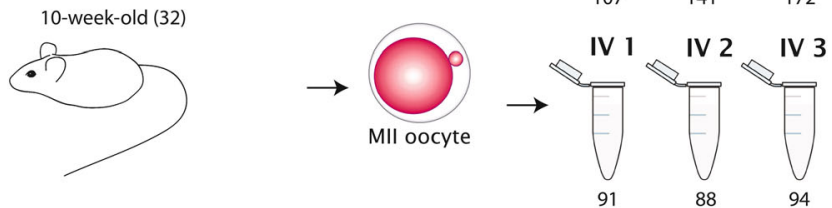

\section{B}

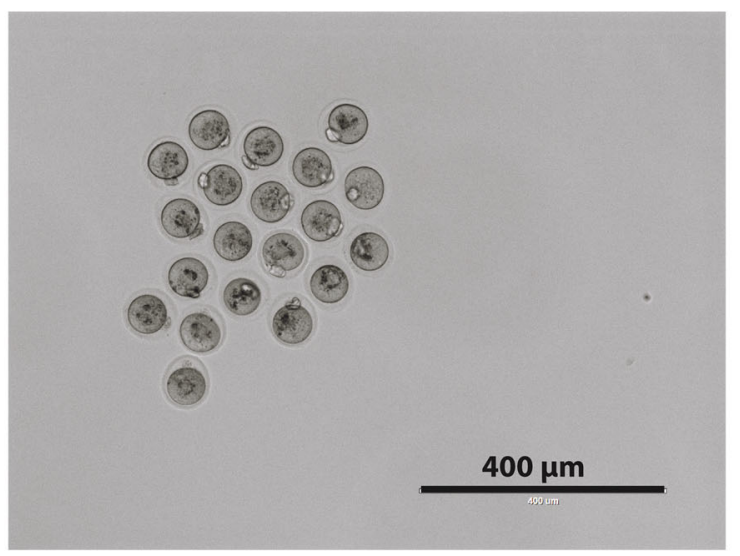

C

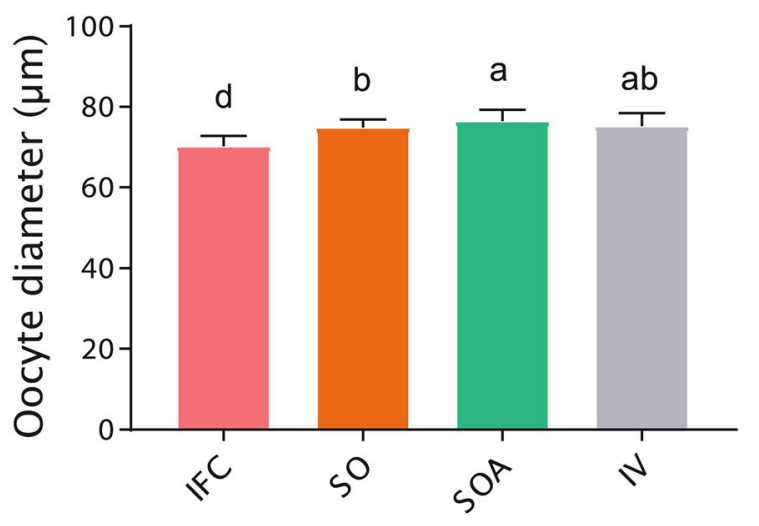

Fig. 1 a Experimental groups used for the genome-wide DNA methylation analysis. The number of females used per group is indicated in brackets next to their age. The tubes represent the number of biological replicates and the number under each tube indicates the number of oocytes pooled in each sample. MII metaphase II, eCG equine chorionic gonadotropin, hCG human chorionic gonadotropin. b MII oocytes from IFC obtained after 10 days of culture. Pictures were taken before snap freezing to measure oocyte diameters. c Oocyte diameter per group. Bar charts show the mean and the standard deviation (SD). Lower-case letters denote significant differences $(p<0.05)$ after applying non-parametric Krustall-Wallis and Dunn's multiple comparisons tests 


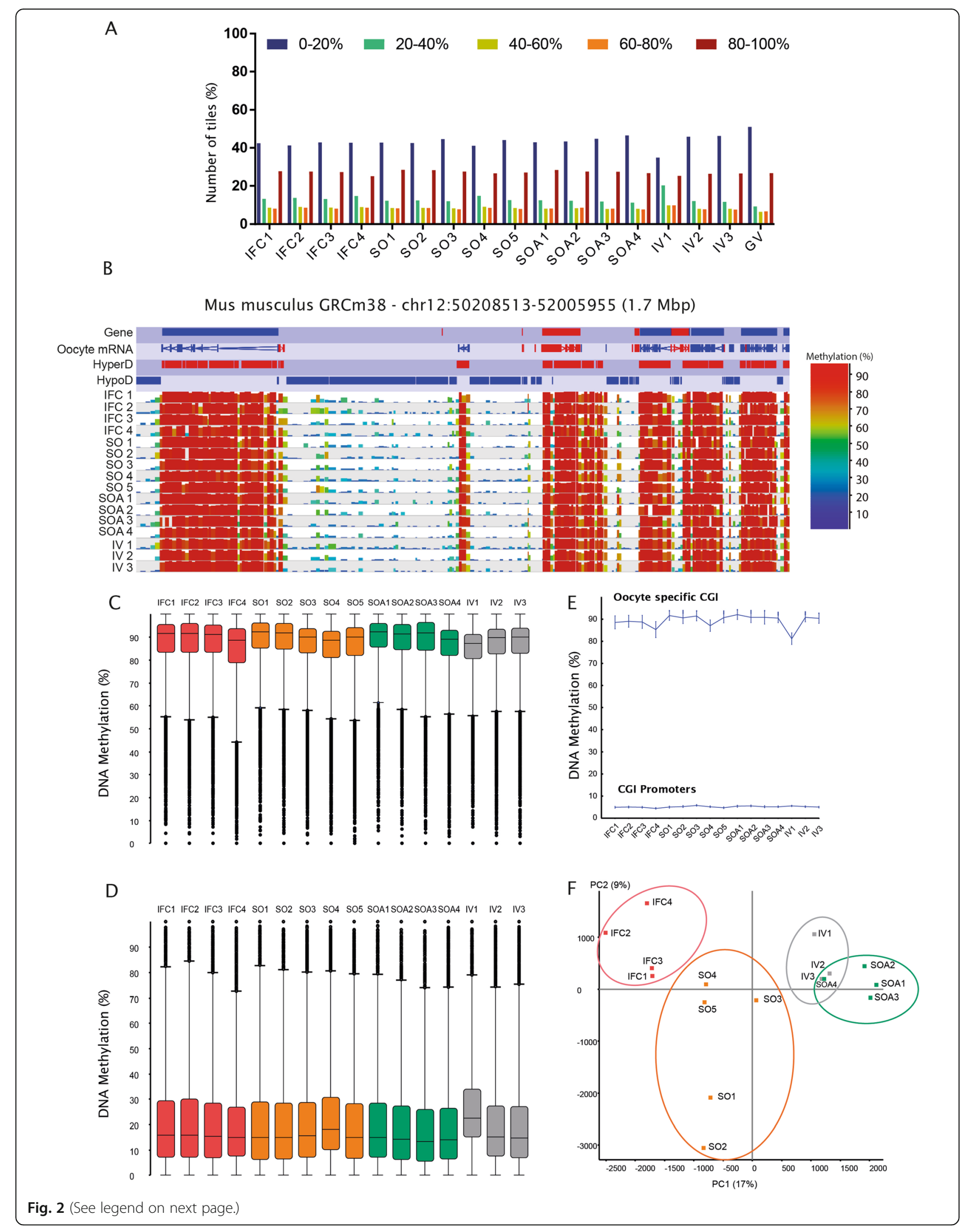


(See figure on previous page.)

Fig. 2 a Distribution of DNA methylation across the genome in 100-CpG windows in all samples compared to Germinal Vesicle (GV) oocytes from Shirane et al. [38]. b SeqMonk screenshot of a $1.7 \mathrm{Mb}$ region of chromosome 11 depicting the hypermethylated (HyperD) and hypomethylated (HypoD) domains characteristic of the oocyte methylome in each of the 16 individual methylation datasets. Genes and oocyte mRNA are shown in red or blue depending on their direction of transcription (forward and reverse, respectively). Each color-coded vertical bar in the screenshot represents the methylation value of a non-overlapping $100 \mathrm{CpG}$ tile. HypoD, HyperD, and oocyte mRNA annotation tracks are derived from Veselovska et al. [24]. c DNA methylation percentages at HyperD in all samples $(n=26,570)$. In the box:whiskers plot, the line across the middle of the box shows the median, the upper and lower extremities of the box show the 25th and 75th percentile of the set of data, and the upper and lower black whiskers show the median plus/minus the interquartile (25-75\%) range multiplied by 2. Individual points which fall outside this range are shown as filled circles, and represent single outlier tiles. $\mathbf{d}$ Box:whisker plot showing the DNA methylation percentages at HypoD $(n=38,739)$. e DNA methylation percentages of CpG Islands (CGI) located at promoters $(n=11,542)$ and CGls highly methylated in oocytes $(n=2014)$. Each point represents the mean value along with error bars indicating the $95 \%$ confidence interval for the measure. $\mathbf{f}$ Principal component analysis (PCA) of informative 100-CpG tiles (value between 0 and 100 in all 16 samples; $n=195,170$ ) shows how biological replicates cluster together within each group and differently between conditions 


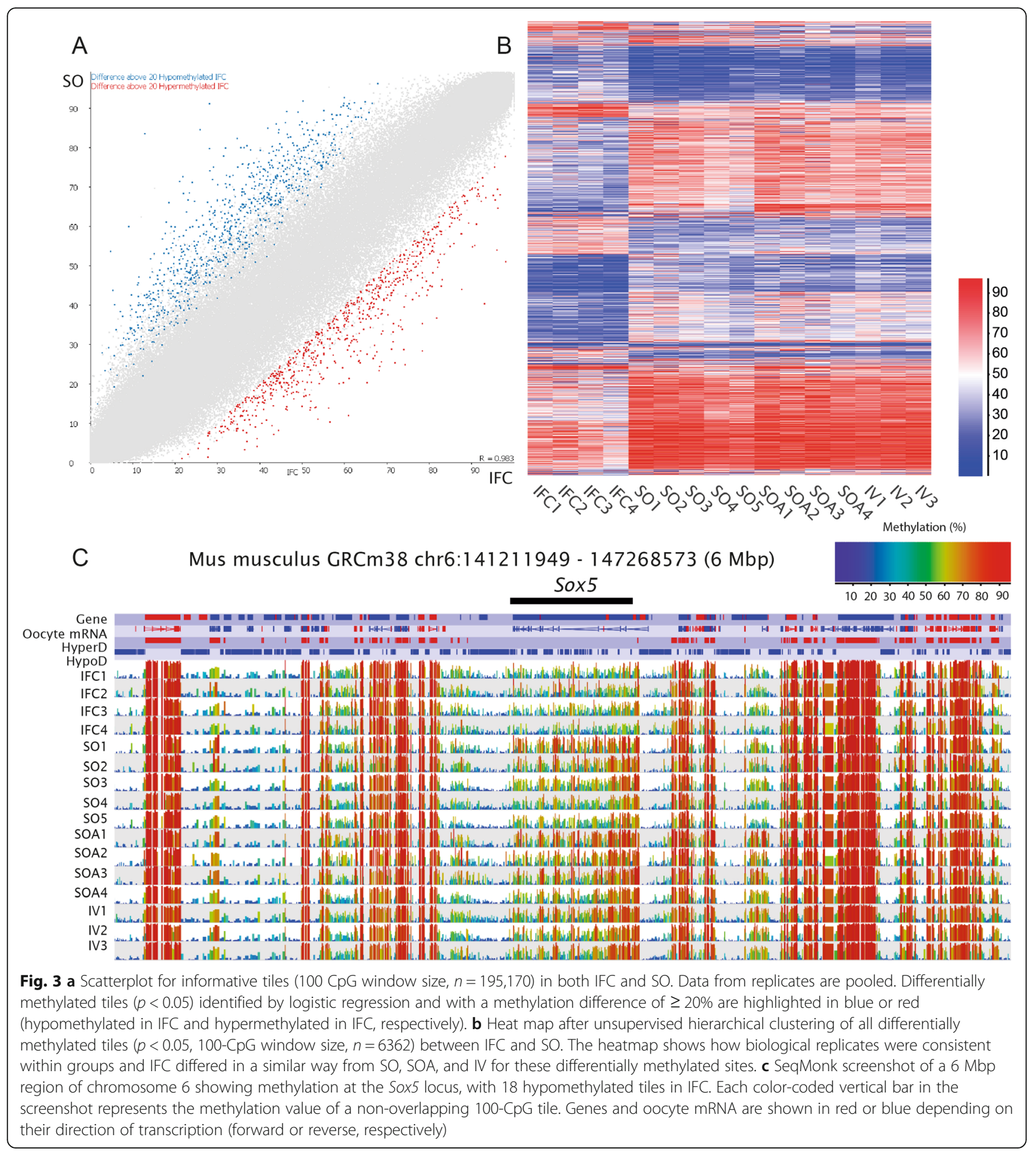




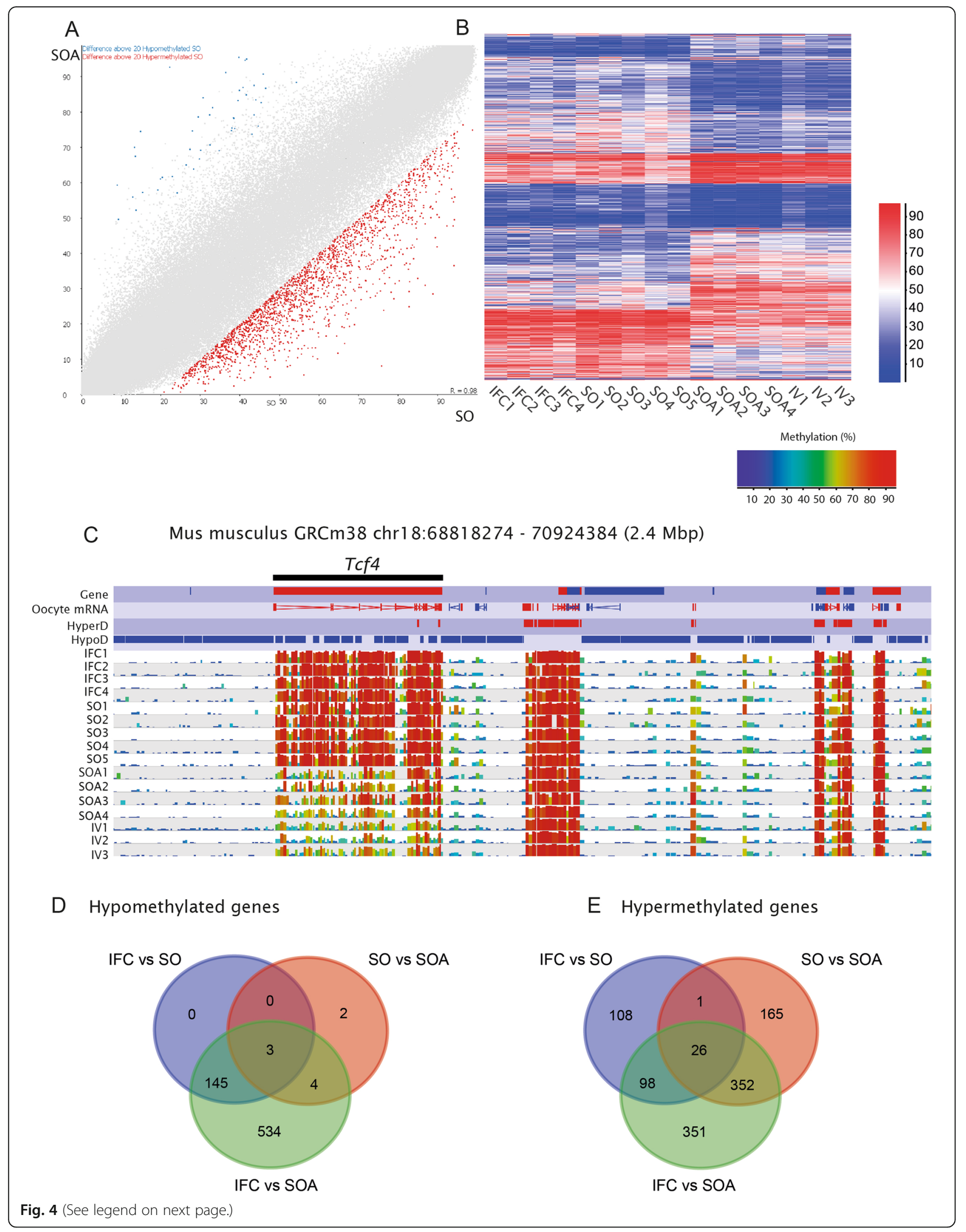


(See figure on previous page.)

Fig. 4 a Scatterplot for common informative tiles (100 CpG window size, $n=195,170$ between SO and SOA. Data from replicates are pooled. Differentially methylated tiles $(p<0.05$ ) identified by logistic regression and with a methylation difference of $\geq 20 \%$ are highlighted in blue or red (hypomethylated in IFC and hypermethylated in IFC, respectively). $\mathbf{b}$ Heat map after unsupervised hierarchical clustering of all differentially methylated tiles $(p<0.05,100 \mathrm{CpG}$ window size, $n=14,795$ between SO and SOA. The heatmap shows that the IFC group followed the same trend as SO, while the IV group was similar to SOA for these differentially methylated sites. c SeqMonk screenshot showing the methylation levels at Tcf4 locus (with 28 hypermethylated tiles). Each color-coded vertical bar represents the methylation value of a non-overlapping 100-CpG tile. Genes and oocyte mRNA are shown in red or blue depending on their direction of transcription (forward or reverse, respectively). $\mathbf{d}_{\text {, e Venn }}$ diagrams showing the common hypomethylated and hypermethylated genes that were affected in IFC vs. SO, SO vs. SOA, and IFC vs. SOA 


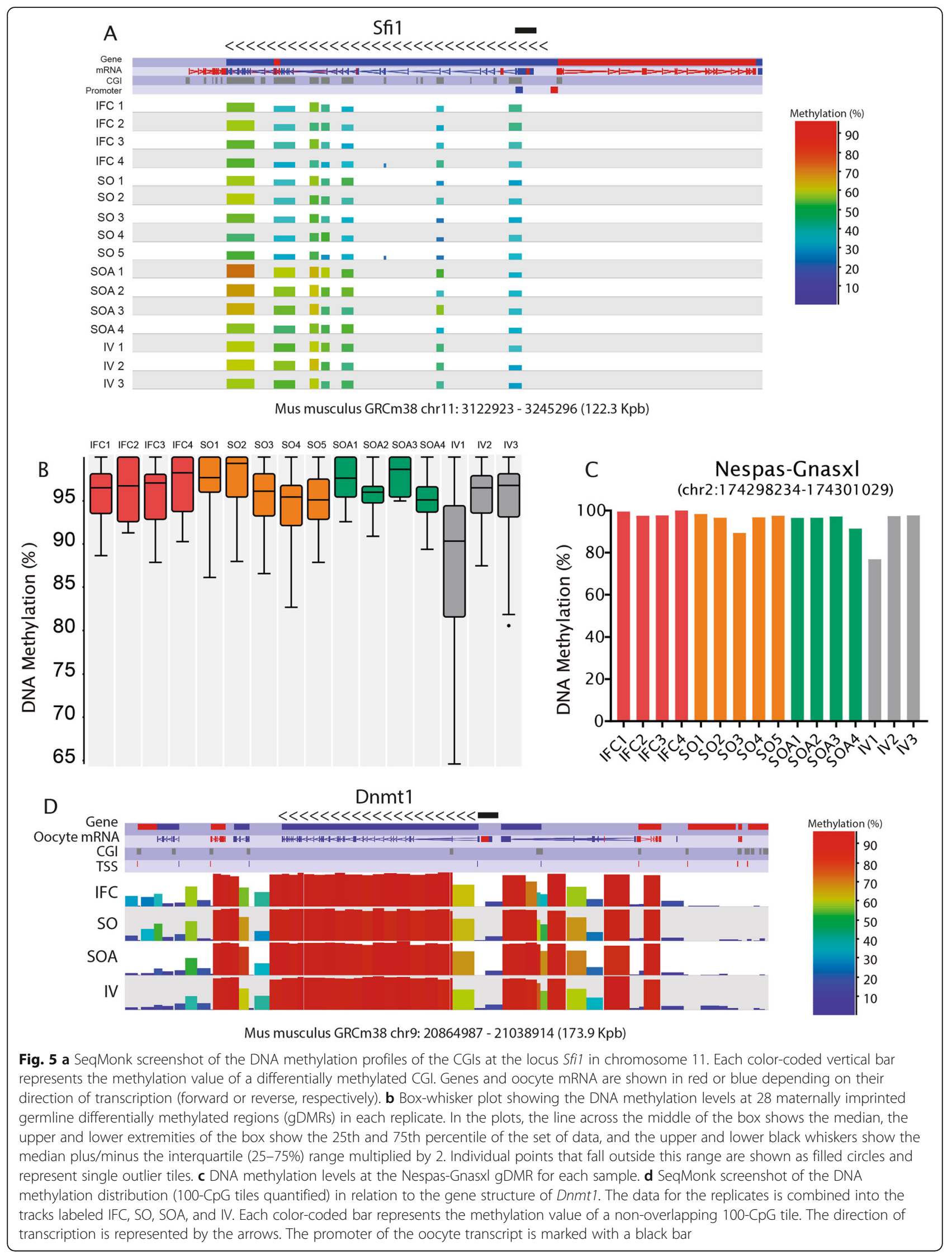

